\title{
The Role of Particle Size in the Dispersion \\ Engineering of Plasmonic Arrays \\ Supporting Information
}

\author{
Veronika Tretnak, Ulrich Hohenester, Joachim R. Krenn, ${ }^{*}$ and Andreas Hohenau \\ University of Graz, Austria
}

E-mail: joachim.krenn@uni-graz.at

\section{Dispersion imaging in the back focal plane}

To verify the opening of a bandgap at normal incidence, we recorded the angle dependent attenuance spectra at a better angular resolution of about $0.4^{\circ}$. To this end we placed the sample in a microscope, reduced the field of view to about $100 \mu \mathrm{m}$ (the size of the particle array) and imaged the objectives back focal plane (diffraction image) directly on the entrance slit of an imaging spectrograph. The resulting images from the spectrograph's camera are calibrated in one dimension in terms of wavelength, in the other in terms of $\sin \theta_{i}$. Like this we can record the angle dependent attenuance spectra as depicted in Fig. S1 (a) and compare them to the angle dependent spectra recorded by a normal microspectrometer when tilting the sample (Fig. S1(b)). In the latter case, the finite aperture of the condensor lens leads to an angular averaging over a range of $\pm 1.6^{\circ}$. The red lines depict the empty lattice dispersion for $500 \mathrm{~nm}$ array period in a homogeneous medium with $n=1.516$. The observed extinction is shifted to lower energies compared to the red lines, as an effect of the ITO layer the particles are situated on. The effect of averaging over a larger angular range in Fig. S1(b) as compared to Fig. S1(a) does qualitatively not affect the main features, i.e. band-splitting and mode brightness.

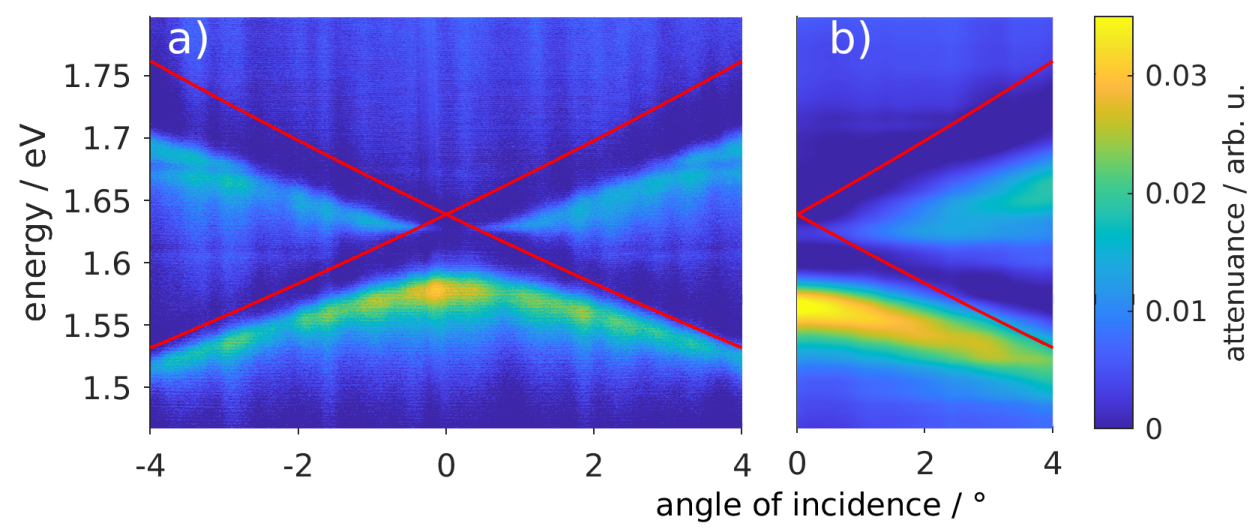

Figure S1: Dispersion relations of $100 \mathrm{~nm}$ long Al nano-rods recorded by (a) imaging the diffraction plane of the array onto the entrance slit of an imaging spectrograph with an angular resolution of about $0.4^{\circ}$, as compared to (b) the spectra recorded in a microspectrometer by tilting the sample with an illumination with $1.6^{\circ}$ divergence, as presented in Fig. 2 of the main text. Red lines depict the empty lattice dispersion for $500 \mathrm{~nm}$ array period in a medium with $n=1.516$. 


\section{Mathematical model of a 1D photonic crystal with and without excitation}

As explained in the main text and as sketched in Fig. S2 we consider a 1D stack of alternating layers of media a and $\mathrm{b}$, with thicknesses $l_{a}=l$ and $l_{b}=d-l$. In every layer, the electric field can be expressed as a superposition of the electric fields of left- and right-propagating electromagnetic waves. At an interface between medium a (refractive index $n_{a}$ ) and medium $\mathrm{b}\left(n_{b}\right)$, the waves are partly reflected and partly transmitted, with an amplitude ratio given by the Fresnel coefficients of the interface $\rho$ and $\tau$, respectively.

a)
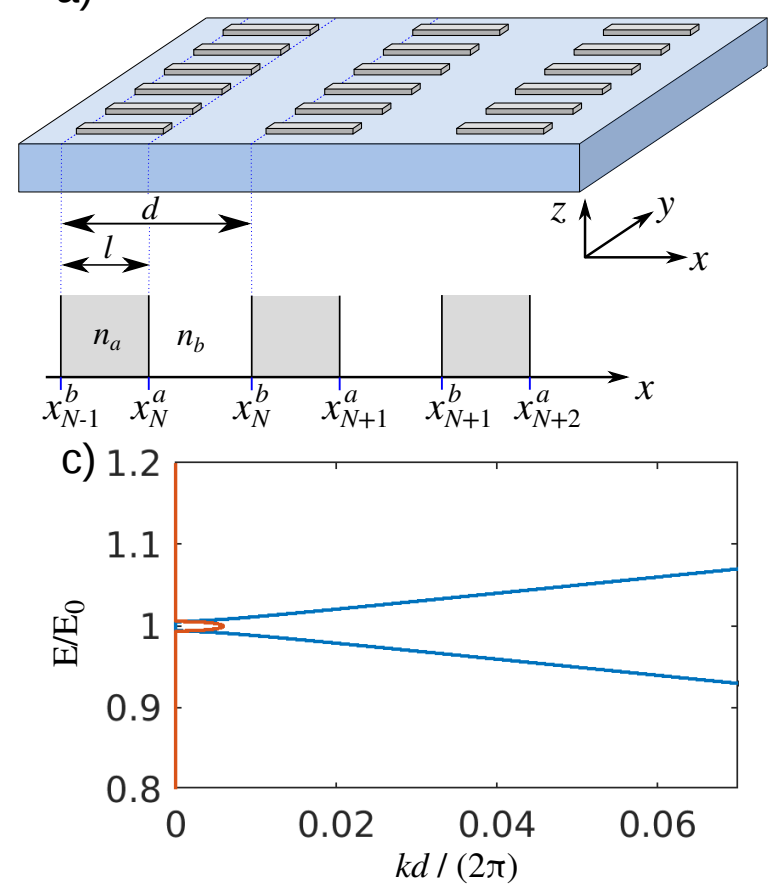
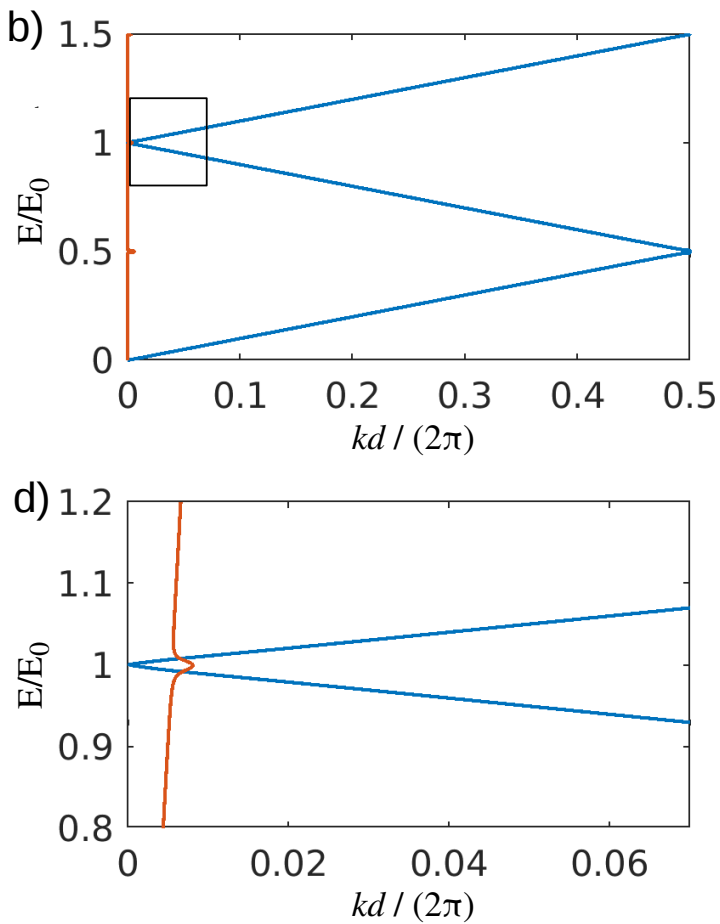

Figure S2: Modelling a regular array (period $d$ ) of nanorods (length $l$ ) on a slab waveguide as a 1D PC by considering $( \pm 1,0)$ CLRs only. (a) The areas occupied by the particles are described by a high refractive index $n_{a}$, while the space in between is assigned a low refractive index $n_{b}$. (b) With $n_{a}=1.516 \times 1.05$, $n_{b}=1.516 \times 1.01$ the $\mathrm{PC}$ dispersion relation closely resembles the empty lattice dispersion, however with some band splitting where the normalized energy $\mathrm{E} / \mathrm{E}_{0}$ is unity. Real and imaginary parts of $k$ are plotted in blue and orange, respectively. (c) Closeup of the rectangluar box in (b). (d) When including damping $\left(n_{a}=1.516 \times(1.05+0.008 i), n_{b}=1.516 \times(1.01+0.005 i)\right)$, the band gap smears out into a region of low propagation length, evidenced by the large imaginary part of $k$ (orange).

In the following we include the excitation of the PC modes from an external wave and consider the electric field amplitudes at the left side of each interface between media a and b. For example, at the left side of the interface at the position $x_{N}^{b}$ (medium a), the amplitude of the right-propagating (superscript + ) wave is a superposition of the right-propagating wave in medium b transmitted by the interface and the part of the left-propagating wave in medium a reflected by the interface at $x_{N}^{a}$. Additionally, we consider that each interface is the source of (somehow externally excited) waves in + and - directions 
with amplitudes $E_{S, N}^{a+}, E_{S, N}^{b+}, E_{S, N}^{a-}$ and $E_{S, N}^{b-}$ :

$$
E_{N}^{b+}=\alpha_{b} E_{N}^{a+} \tau_{a b}+\alpha_{b}^{2} E_{N}^{b-} \rho_{b a}+\alpha_{b} E_{S, N}^{a+} .
$$

Here, $\alpha_{b}=e^{i n_{b} k_{0} l_{b}}$ is the phase retardation a wave experiences by passing through the layer $\mathrm{b}$, with $k_{0}=\omega / c$ being the vacuum wave number and $l_{b}=l-d$ being the thickness of layer $\mathrm{b}$. Accordingly, the left-propagating wave at the left side of the interface at $x_{N}^{a}$ (medium b) can be written as:

$$
E_{N}^{a-}=\alpha_{b} E_{N}^{b-} \tau_{b a}+E_{N}^{a+} \rho_{a b}+E_{S N}^{a-}
$$

From these two equations we can express $E_{N}^{b+}$ and $E_{N}^{b-}$ :

$$
\left(\begin{array}{l}
E_{N}^{b+} \\
E_{N}^{b-}
\end{array}\right)=\mathbf{M}_{b}\left(\begin{array}{l}
E_{N}^{a+} \\
E_{N}^{a-}
\end{array}\right)+\mathbf{M}_{S b}\left(\begin{array}{c}
E_{S, N}^{a+} \\
E_{S, N}^{a-}
\end{array}\right)
$$

with

and

$$
\mathbf{M}_{b}=\left[\begin{array}{cc}
\alpha_{b} \tau_{a b}-\frac{\alpha_{b} \rho_{b a} \rho_{a b}}{\tau_{b a}} & \frac{\alpha_{b} \rho_{a b}}{\tau_{b a}} \\
-\frac{\rho_{a b}}{\alpha_{b} \tau_{b a}} & \frac{\tau_{b} \tau_{b a}}{\alpha_{b}}
\end{array}\right]
$$

Similarly, one can derive

$$
\mathbf{M}_{S b}=\left[\begin{array}{cc}
\alpha_{b} & -\frac{\alpha_{b} \rho_{b a}}{\tau_{b a}} \\
0 & -\frac{1}{\alpha_{b} \tau_{b a}}
\end{array}\right]
$$

$$
\left(\begin{array}{l}
E_{N+1}^{a+} \\
E_{N+1}^{a-}
\end{array}\right)=\mathbf{M}_{a}\left(\begin{array}{l}
E_{N}^{b+} \\
E_{N}^{b-}
\end{array}\right)+\mathbf{M}_{S a}\left(\begin{array}{c}
E_{S, N}^{b+} \\
E_{S, N}^{b-}
\end{array}\right)
$$

with

and

$$
\mathbf{M}_{a}=\left[\begin{array}{cc}
\alpha_{a} \tau_{b a}-\alpha_{a} \frac{\rho_{a b} \rho_{b a}}{\tau_{a b}} & \frac{\alpha_{a} \rho_{b a}}{\tau_{a b}} \\
-\frac{\rho_{b a}}{\alpha_{a} \tau_{a b}} & \frac{1}{\alpha_{a} \tau_{b a}}
\end{array}\right]
$$

By inserting Eq.1 into Eq. 2 we get

$$
\mathbf{M}_{S a}=\left[\begin{array}{cc}
\alpha_{a} & -\frac{\alpha_{a} \rho_{a b}}{\tau_{\tau b}} \\
0 & -\frac{1}{\alpha_{a} \tau_{a b}}
\end{array}\right] .
$$

$$
\left(\begin{array}{c}
E_{N+1}^{a+} \\
E_{N+1}^{a-}
\end{array}\right)=\mathbf{M}_{a} \mathbf{M}_{b}\left(\begin{array}{c}
E_{N}^{a+} \\
E_{N}^{a-}
\end{array}\right)+\mathbf{M}_{a} \mathbf{M}_{S b}\left(\begin{array}{c}
E_{S, N}^{a+} \\
E_{S, N}^{a-}
\end{array}\right)+\mathbf{M}_{S a}\left(\begin{array}{c}
E_{S, N}^{b+} \\
E_{S, N}^{b-}
\end{array}\right),
$$

which can be formally rewritten as

$$
\left(\begin{array}{l}
E_{N+1}^{a+} \\
E_{N+1}^{a-1}
\end{array}\right)=\mathbf{M}\left(\begin{array}{l}
E_{N}^{a+} \\
E_{N}^{a-}
\end{array}\right)+\left(\begin{array}{l}
E_{S}^{+} \\
E_{S}^{-}
\end{array}\right) \stackrel{!}{=} \beta\left(\begin{array}{l}
E_{N}^{a+} \\
E_{N}^{a-}
\end{array}\right) .
$$

The right side of the equation takes into account that the electric fields have to be lattice-periodic functions with a complex phase factor $\beta=e^{i k d}$.

To solve this equation, we look at two cases: the free and the excited lattice.

- For the free case, $E_{S}^{+}=E_{S}^{-}=0$. Then, Eq. 3 reduces to

$$
\mathbf{M}\left(\begin{array}{c}
E_{N}^{a+} \\
E_{N}^{a-}
\end{array}\right)=\beta\left(\begin{array}{c}
E_{N}^{a+} \\
E_{N}^{a-}
\end{array}\right) .
$$

$\beta$ are the Eigenvalues of $\mathbf{M}$, from which the complex wave number $k$ of the corresponding Eigenmode can be calculated as a function of frequency. This leads to the dispersion relations as depicted in Fig. S2(b). 
- For the excited case, Eq. 3 can be rewritten as

$$
\left(\begin{array}{l}
E_{N}^{a+} \\
E_{N}^{a-}
\end{array}\right)=(\mathbf{M}-\mathbb{I} \beta)^{-1}\left(\begin{array}{c}
E_{S}^{+} \\
E_{S}^{-}
\end{array}\right),
$$

i.e. the strengths of the left- and right-propagating waves can be determined from the source vector. Here, $\beta=e^{i k_{x} d}$ with $k_{x}$ corresponding to the wave vector $x$-component of the exciting plane wave in the experiment. For the source vector, we made further simplifications: The strength of the left- and right-emitted waves are identical and independent of the interface, i.e. $\left|E_{S, N}^{a+}\right|=\left|E_{S, N}^{b+}\right|=$ $\left|E_{S N}^{a-}\right|=\left|E_{S, N}^{b-}\right|=0.1 E_{0}$, with $E_{0}$ being the amplitude of the exciting wave. What remains is the phase factor of the source terms, which is determined by the exciting wave as $e^{i k_{x} x}$ with $x$ being the $x$-coordinate of the respective interface at $x_{N}^{a}$ or $x_{N}^{b}$, and a factor of \pm 1 depending on the emission into either a high- or a low index region. For calculating of Fig. 8 in the main text we plot the quantity $\left|E_{N}^{a+}+E_{N}^{a-}+E_{0}\right|^{2}$ (which is porpotional to the square of the polarization of the particle regions and thus the absorbance) and presume its correspondence to the exerimentally accessible attenuance.

\section{Approximating the field energy of 1D PC modes}

We assume the fields $E=E_{0} \cos k x$ for the symmetric mode and $E=E_{0} \sin k x$ for the antisymmetric mode and low refractive index differences so that $k$ is independent of the material and the field energy is proportional to $E^{2}$. Then the field energy in the high- $n$ region of the nanorods of length $l$ is

$$
E_{a}=\int_{-l / 2}^{l / 2} \frac{1}{2}\left(1 \pm \cos \left(2 \frac{x}{d} 2 \pi\right) \mathrm{d} x=\frac{1}{2} \frac{d}{2 \pi}\left[l \frac{2 \pi}{d} \pm \sin \left(l \frac{2 \pi}{d}\right)\right]\right.
$$

For the expression of the field energy in the low- $n$ region $E_{b}, l$ has to be replaced by $d-l$. The plus sign is for the symmetric mode, the minus sign is for the antisymmetric mode. 


\section{Comparison to non-metallic particles}

The size dependence of the CLR branch brightness is not limited to metal particles but can be observed in regular arrays built from any material, provided that the CLRs are strong enough to be observed. As an example, we compare square arrays $(d=500 \mathrm{~nm})$ of identically shaped nanorods (width $80 \mathrm{~nm}$, height $30 \mathrm{~nm}$, lengths 200 or $300 / 400 \mathrm{~nm}$ ) of either aluminum (Fig. S3(a)) or indium tin oxide (ITO) (Fig. S3(b)). We observe two peaks, corresponding to the high energy and low energy branches of the CLR dispersion. Clearly, for $l=200 \mathrm{~nm}$ the low energy peak is dominating (i.e. bright), whereas for $l=300 \mathrm{~nm}(\mathrm{Al})$ or $400 \mathrm{~nm}$ (ITO) the high energy peak is stronger, as marked by the diamond and circle symbols. We note that the finite visibility of both branches in the spectra is due to the angular detection range in our optical setup that leads to angular integration over the CLR dispersion.

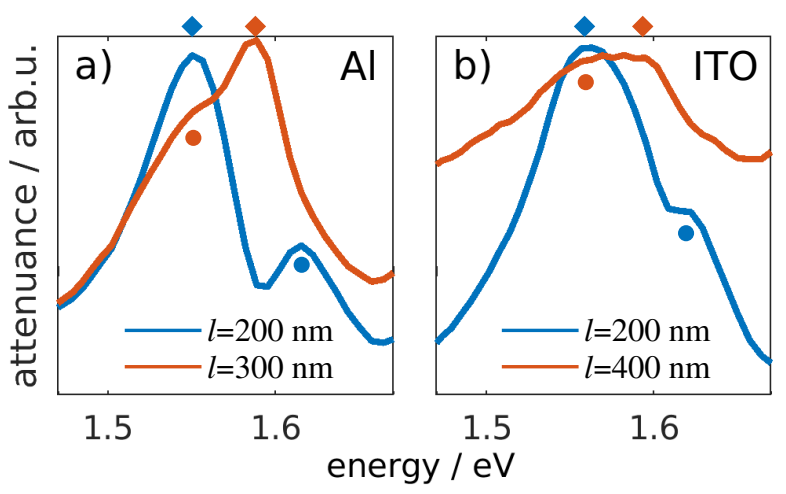

Figure S3: Measured attenuance spectra of square arrays $(d=500 \mathrm{~nm})$ of (a) aluminum and (b) ITO nanorods of $80 \mathrm{~nm}$ width, $30 \mathrm{~nm}$ height and lengths of (blue) $200 \mathrm{~nm}$ and (orange) $300 \mathrm{~nm}$ (aluminium) or $400 \mathrm{~nm}$ (ITO). The upper blue and orange diamonds mark the spectral position of the bright CLR peaks, the circles point out the darker CLR peaks that are still visible for both rod lengths due to the finite angular detection range of the used optics. 